\title{
Calibration uncertainty in ocean color satellite sensors and trends in long-term environmental records
}

\author{
Kevin R. Turpie*a, Robert E. Eplee, Jr. ${ }^{\mathrm{b}}$, Bryan A. Franz ${ }^{\mathrm{c}}$, Carlos Del Castillo ${ }^{\mathrm{c}}$ \\ ${ }^{a}$ University of Maryland, Baltimore County, Joint Center for Earth Systems Technology 5523 \\ Research Park Dr. \#320, Baltimore, MD, USA 21228; 'Science Applications International \\ Corporation, Beltsville, Maryland, USA 20705; 'NASA Goddard Space Flight Center, Greenbelt, \\ Maryland 20771, USA
}

\begin{abstract}
Launched in late 2011, the Visible Infrared Imaging Radiometer Suite (VIIRS) aboard the Suomi National Polar-orbiting Partnership (NPP) spacecraft is being evaluated by NASA to determine whether this sensor can continue the ocean color data record established through the Sea-Viewing Wide Field-of-view Sensor (SeaWiFS) and the MODerate resolution Imaging Spectroradiometer (MODIS). To this end, Goddard Space Flight Center generated evaluation ocean color data products using calibration techniques and algorithms established by NASA during the SeaWiFS and MODIS missions. The calibration trending was subjected to some initial sensitivity and uncertainty analyses. Here we present an introductory assessment of how the NASA-produced time series of ocean color is influenced by uncertainty in trending instrument response over time. The results help quantify the uncertainty in measuring regional and global biospheric trends in the ocean using satellite remote sensing, which better define the roles of such records in climate research.
\end{abstract}

Keywords: VIIRS, remote sensing, phytoplankton, ocean color, calibration, uncertainty, time series, climate data record

\section{INTRODUCTION}

Launched in late 2011, the Visible Infrared Imaging Radiometer Suite (VIIRS) aboard the Suomi National Polar-orbiting Partnership (NPP) spacecraft is being evaluated by NASA to determine whether this sensor can continue the ocean color data record established through the Sea-Viewing Wide Field-of-view Sensor (SeaWiFS) and the MODerate resolution Imaging Spectroradiometer (MODIS). To this end, the NASA Goddard Space Flight Center (GFSC) generated evaluation ocean color data products using calibration techniques and algorithms established by NASA during the SeaWiFS and MODIS missions. The calibration trending was subjected to sensitivity and uncertainty analyses. Of particular interest was, and continues to be, the effect of calibration trending uncertainties on derived ocean color data products, such as chlorophyll $a$ concentration, which is used to monitor phytoplankton activity at synoptic scales.

Phytoplankton are estimated to contribute to as much as half of global primary productivity, essentially all marine productivity, and support nearly all major aquatic food webs. Understanding and quantifying global changes in phytoplankton communities in the ocean is important to linking ecological and biogeochemical responses to climate change. Satellite chlorophyll $a$ concentration, $C h l a$, as derived using the OC3 algorithm [1], is a standard data product being implemented for the VIIRS instrument. This standard product is currently the principal indicator of phytoplankton activity in the upper layers of the ocean. This is also true for other instruments on which the NASA climate data record is based, including MODIS onboard the Aqua spacecraft (MODIS Aqua) and SeaWiFS. The analogous algorithm for SeaWiFS is OC4, which makes use of an additional blue band at $510 \mathrm{~nm}$ [1]. OC3 computes Chl $a$ as a exponentiallinear function of green to blue band ratios, more specifically the maximum of $R_{r s}(551 \mathrm{~nm}): R_{r s}(443 \mathrm{~nm})$ and $R_{r s}(551 \mathrm{~nm}): R_{r s}(488 \mathrm{~nm})$, where $R_{r s}(\lambda)$ is the remote sensing reflectance for the ocean surface at the band centered at wavelength $\lambda$. $R_{r s}(\lambda)$ is derived from the VIIRS radiometry above the atmosphere through the removal of the light contributed by the atmosphere. Because the atmospheric contribution to the at-sensor light level can be over $90 \%$, small errors in the top-of-atmosphere (TOA) measurements are large relative to the desired contribution from the surface. In addition, measurements in the red band and two NIR bands factor into the calculation of the atmospheric contribution. Thus, Chl $a$ is influenced by uncertainties in the four aforementioned visible bands and two NIR bands (six of the seven VIIRS ocean bands).

*kevin.r.turpie@nasa.gov; phone 301 286-9996; fax 301 286-0268; oceancolor.gsfc.nasa.gov 
Here we present an introductory assessment of how the NASA-produced time series of ocean color is influenced by uncertainty in trending instrument response over time. Work in on this problem will help quantify the uncertainty in measuring regional and global biospheric trends in the ocean using satellite remote sensing, which better define the roles of such records in climate research. Included is an initial evaluation of potential uncertainty stemming from calibration trend errors and directions for further work.

\section{BACKGROUND}

Much work has been done to quantify trends in the existing chlorophyll record [2, 3], including focusing on the existing decade and a half of global satellite data [4,5]. Many efforts to extract a trend in chlorophyll show a downward trend, and expanding oligotrophic regions, across all ocean basins [6,7]. A corresponding increase in eutrophic regions has also been noted, including coastal regions [8]. Models of primarily productivity that are dominated by the satellite $\mathrm{Chl} a$ likewise saw distinct trends, [9]. Intuitive physical explanations have been suggested for these trends. For instance, the decrease in productivity in the ocean gyres could stem from acidification of ocean waters or the rise in global sea surface temperature. Conversely, this process might be countered and overtaken by anthropogenic eutrophication, increasing Chl $a$ in some coastal waters. However, considerable debate has been raised regarding the validity of the some of observed trends $[10,11]$. Some noted a number of inconsistencies in the reports. Others simply reported more mixed results $[12,13]$ or noted the insufficient length of the satellite data record $[14,15]$. Siegel et al. [11] did note the importance of highly accurate instrument calibration for assessing global changes in Chl a, clearly pointing in an important direction that was not being discussed. None of these chlorophyll trend studies considered the contribution of
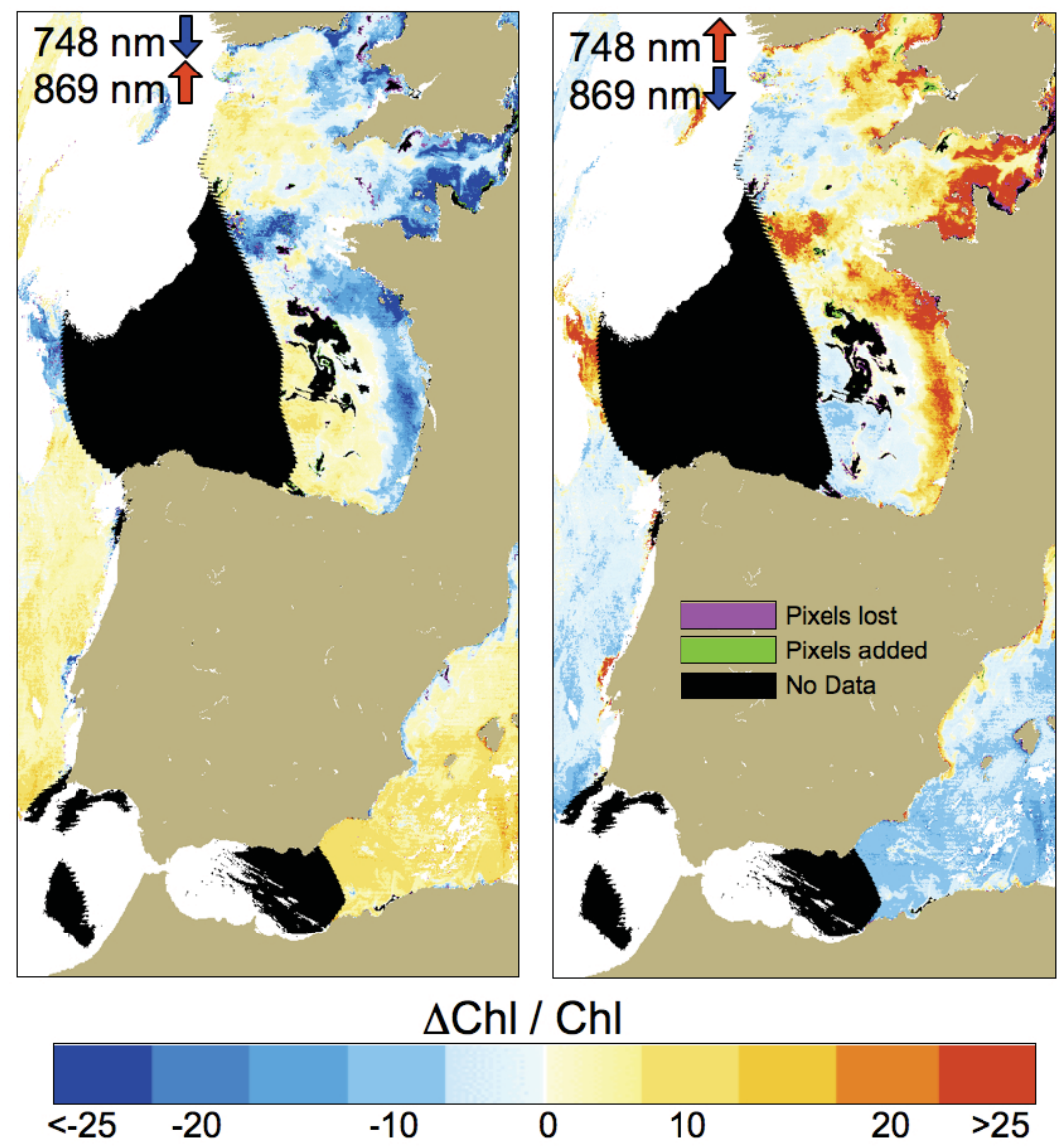

Figure $\mathbf{1}$ - Small changes in calibration lead to big changes in $\mathbf{C h l} \boldsymbol{a}$. The image taken from MODIS Aqua shows the effect of perturbing the NIR calibration $0.3 \%$ in opposite directions between the two bands. The $20 \%$ percent or more change in Chl $a$ is substantial. It is also interesting that the eutrophic coastal waters and lower concentrations of more open water respond oppositely [16]. 
small instrument calibration drifts.

An earlier sensitivity analysis of ocean color algorithms demonstrated that calibration changes of only a small fraction of a percent in the Near Infrared (NIR) bands of ocean color instruments could cause decreasing low chlorophyll $a$ measurements and increasing high chlorophyll measurements [16]. The phenomenon seen in Figure 1 shows the effect on high and low chlorophyll $a$ values for opposite changes in the 750 and $865 \mathrm{~nm}$ bands of $0.3 \%$ (the criterion threshold for the calibration stability requirement for S-NPP VIIRS). In the right hand example, the small perturbations in the NIR calibration caused higher coastal concentrations to increase, while lower open waters concentrations to decreased. These changes in Chl $a$ are greater than $20 \%$ and far exceed the amounts noted in studies based on satellite ocean color data. The NIR bands are used to help remove of the aerosol component of the atmospheric signal in order to obtain $R_{r s}(\lambda)$, which is used to calculate $C h l a$. Although this portion of the atmospheric correction is only about $20 \%$ of the total TOA signal being removed, it is particularly subject to error and hence represents a major source of uncertainty in computing $R_{r s}(\lambda)$ for the visible wavelengths. Likewise, an error in the calibration of each visible band can lead to an order of magnitude larger error in the corresponding value for $R_{r s}(\lambda)$. Figure 2 shows the relative $R_{r s}(\lambda)$ error resulting from small errors in calibration [16]. Meanwhile, the standard calculation for Chl a dependent on $R_{r s}(\lambda)$ ratios of green band $(551 \mathrm{~nm})$ to blue bands $(443$ or $488 \mathrm{~nm})$. Because the aerosol correction tends to affect the denominator of these ratios differently than the numerator, the subsequent error in the chlorophyll algorithm can be quite large.

Given that $C h l a$ is sensitive to very small changes in instrument calibration, it is clear that the effect of any potential changes to the instrument with time on Chl a must be quantified before credible statements can be made regarding the Chl $a$ trend. This study begins with investigation of very small, uncorrected drift in the on-orbit calibration, and is primarily based on known residuals observed in trending instrument changes. Estimating the size of instrument calibration drift residuals on the quality of the NASA climate data record will help refine our understanding of this

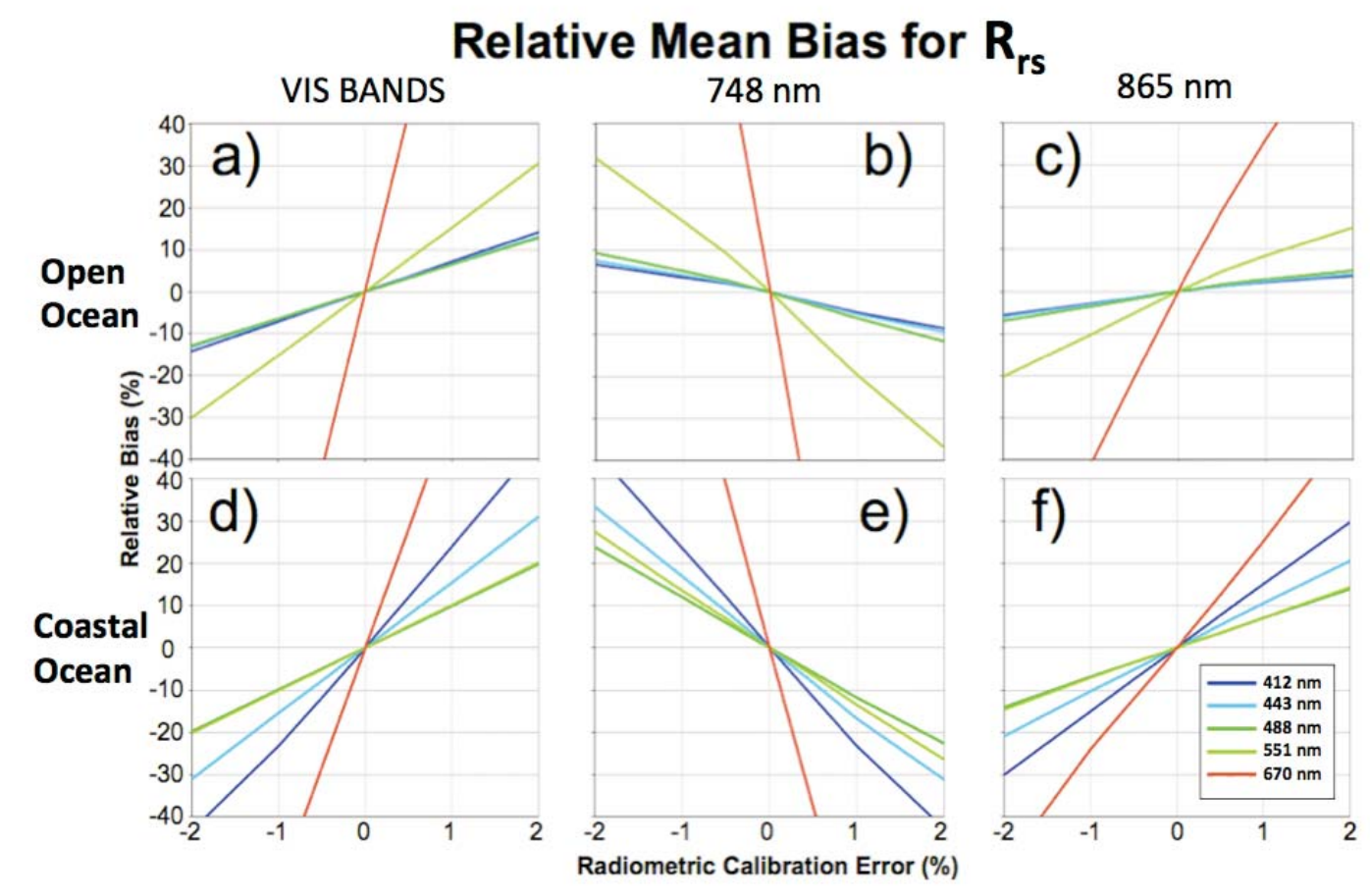

Figure 2 - Sensitivity of $\boldsymbol{R}_{r s}(\lambda)$ to small errors in calibration. The ordinate represents changes in $R_{r s}(\lambda)$ in response to changes in calibration, which are given along the abscissa. Calibration errors directly affect the top-ofatmosphere measurements, and lead to large changes in $R_{r s}(\lambda)$. The top row was taken from an open ocean MODIS scene (a-c) and the bottom row likewise uses data from a coastal scene (d-f). Small calibration changes in any visible band results directly in a large $R_{r s}(\lambda)$ change for the corresponding band (a,d). Likewise, changes in each of the NIR band calibration leads to a similar sized error (b,c,e,\&f) in all visible bands. The response in $R_{r s}(\lambda)$ varies between bands and between ocean and coastal water because the underlying $R_{r s}$ values differ between bands and geographic location. [16] 
influence. In addition, this will help to us understand how to investigate these effects for MODIS and SeaWiFS.

Ocean color instruments are calibrated prior to launch using NIST-traceable laboratory sources to within $2 \%$ uncertainty for TOA reflectance measurements. The instrument is also designed to facilitate calibration stability of $0.3 \%$ for onorbit calibration. Changes in the instrument calibration are monitored using either an onboard Spectralon ${ }^{\mathrm{TM}}$ panel, called the solar diffuser (SD), or the moon. These trends are subsequently used to correct the conversion of dark count subtracted digital numbers to TOA spectral radiance in physical units (e.g., $\mathrm{W} \mathrm{m}^{-2} \mathrm{~nm}^{-1} \mathrm{sr}^{-1}$ ) or reflectance (in units of $\mathrm{sr}^{-1}$ ). The latter is obtained by dividing the TOA radiance by the extraterrestrial solar irradiance [17]. In the terminology associated with VIIRS, these correction factors are typically denoted as $F$.

Figure 3 shows the dramatic change in the red and especially the NIR bands, which are substantially larger than the VIIRS blue bands (or than any band on MODIS Aqua). This is because of contamination of four telescope mirrors with a tungsten oxide compound during their manufacture. When exposed to the intense UV light of the space environment, this compound decreases mirror reflectivity (and hence the instrument responsivity) in the red to shortwave IR (SWIR) wavelengths. Thus, a substantially large relative change in these bands must be accurately trended and corrected. Under some circumstances, this could possibly negatively affect the accuracy of the NIR and red trending.

In addition, the residuals of the fits for all bands show both a largely uncorrelated noise component and systematic, seasonal variation. The seasonal nature of these artifacts can be seen in the variation around the fits for the blue bands in Figure 3. These artifacts are of similar size and nature for the red and NIR bands, but less periodic (e.g., different or additional systematic sources may be at play). Both limit the size of any residual spurious trend that may be included the calibration trend, and hence the standard data products. However, the effect of the systematic seasonal component is much larger than the uncorrelated noise. It is currently believed amongst the VIIRS calibration teams that this seasonal variation in the residuals is caused by biases in instrument calibrator system characterization look-up tables. These do not represent actual changes in the response of VIIRS and they may be improved with time with further analysis. This is also supported by the fact that no systematic seasonal fluctuation has been observed the lunar time series (after correcting for libration effects in the RObotic Lunar Observatory or ROLO model) [17]. Therefore, quantification of spurious trends will need to be reassessed with any future improvements in quantification and removal of these

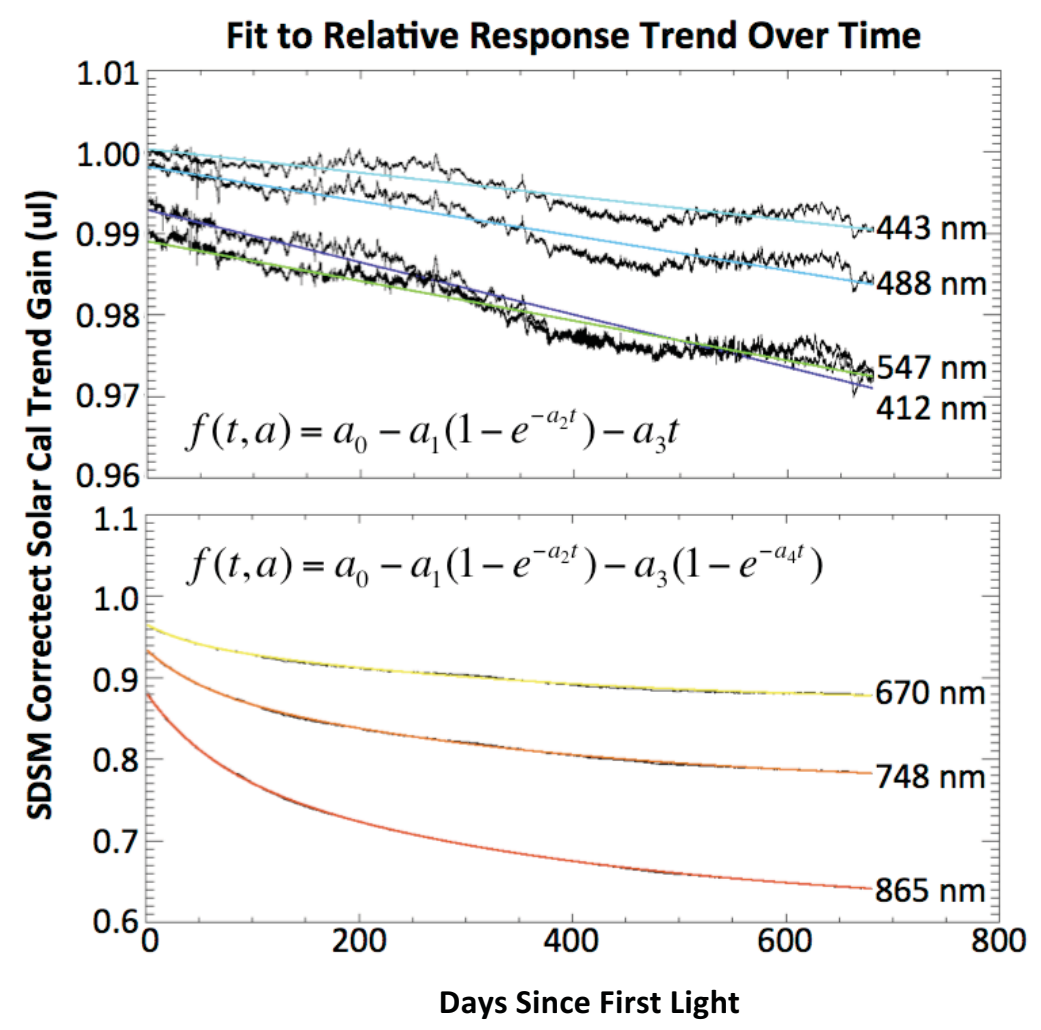

Figure 3 - Solar calibration trending. Two different functional forms are used for different sets of bands as indicated by the formulas given. The resulting gain trends are equal to the inverse of the SD trending factor, $F$. 
systematic artifacts in the residuals.

The accuracy of this trending approach in representing the true change in the instrument response is limited by, in order of process, four sources of uncertainty: 1) the ability of the selected functional form to describe the actual change, 2) random and systematic variability in the SD measurement time series that affect the fitting process, 3) the sample size and the length of the time series, and 4) periodic extrapolation of calibration trending. In this study, we investigated the size of underlying spurious variation that stems from the second source. Others will be explored in subsequent work. Once all these sources are evaluated, their output can then be tied to sensitivity analyses of the ocean color algorithms to quantify the resulting change in time series of $R_{r s}(\lambda)$ and subsequently $C h l a$.

\section{METHODOLOGY}

Because of the complexity of the fit employed by NASA, Monte Carlo techniques were employed to estimate the effect of the uncertainty source stemming from random variation in the trend data. To address the effects of variability, the functional fits that were originally calculated for each band were assumed to be the "true" instrument response trend (as determined using the SD). In this Monte Carlo experiment, many repeated fits are made to these "true" trends plus a noise model. The noise model consists of, at most, two components: one for white noise (i.e., Gaussian distributed and uncorrelated) and one systematic, seasonal curve (i.e., a sinusoid). The analysis initially looked at modeling white noise alone and then a seasonal component was added later. The combination of the "true" trend and the noise model is the modeled data. The fit to the modeled data will be referred to as the modeled trend. The difference between the "true" trend and the modeled trend will be hence called the modeled spurious trend. For each trial in the Monte Carlo experiment, many instances of the modeled spurious trend are generated.

For each trial, the modeled noise was randomized as to not reproduce the fit residual, but vary in a similar fashion. Otherwise, the results will be underestimated because the original curve would be reproduced and hence the residual would be minimized. With each instance of the modeled data, the white noise component is resampled and the systematic component is varied uniformly in phase and amplitude. Improvements on this scheme may be effected as the residual is further analyzed. In initial Monte Carlo experiments, the maximum range for the spurious trends are on the order of $0.05-0.3 \%$ over the first two years of the VIIRS mission. This represents a potentially significant effect in the $R_{r s}$ and $\mathrm{Chl} a$ trends and further emphasized the need for this study. As an example, Figure 4 shows the difference curves (i.e., modeled spurious trends) for a single trial.

\section{RESULTS}

The modeled spurious trends in Figure 4 were generated with Gaussian white noise with a quarter percent standard deviation and with no seasonal variation. Only sixteen points were used as a test, but the actual experiment used the tens of thousands points corresponding to the actual SD time series. The results for this single trial interestingly (but probably coincidentally) show the NIR bands as being anti-correlated and varying as much as $0.3 \%$. The expected influence on chlorophyll concentrations where the maximum NIR differences occur would be similar to the results shown in Figure 1 (not counting the effect of biases in visible bands). It is also interesting to note that the resulting variation is highly auto-correlated, as opposed to the input noise. Thus the resulting modeled spurious trends could induce a small secular trend in the time series of the calibrated satellite data, even though no significant secular trend exists in the input noise model.

Further trials were generated (10 to 100 thousand) and the root mean square error (RMSE) for each modeled spurious trend was taken. This process was repeated for a range of different modeled noise levels. The results showed that there is a one-to-one correspondence between input white noise and the RSME of the modeled spurious trends. The Gaussian component of noise observed in NASA's operational fits to the instrument trending (see Figure 3) is within the 0.05 to $0.1 \%$ range. Thus, the white noise component alone should have much less influence on estimates of Chl $a$ than shown in Figure 1. However, the impact could still be significant and bring into question any claimed observation of synopic variation of Chl $a$ on the order of few percent over the first two years of the mission.

The observed residuals also show a large seasonal component at around the $0.1 \%$ level. When a sinusoidal component with $0.1 \%$ amplitude was added to the noise model in the Monte Carlos experiment the resulting RSME of the modeled spurious trend was again very close to the noise amplitude. Thus, this component of the noise contributes to the majority of the chlorophyll $a$ trend uncertainty. Even thought this spurious signal in the instrument response monitoring is removed from by the functional fit, its influence on the fit accuracy (i.e., the ability to recover the underlying "true" 


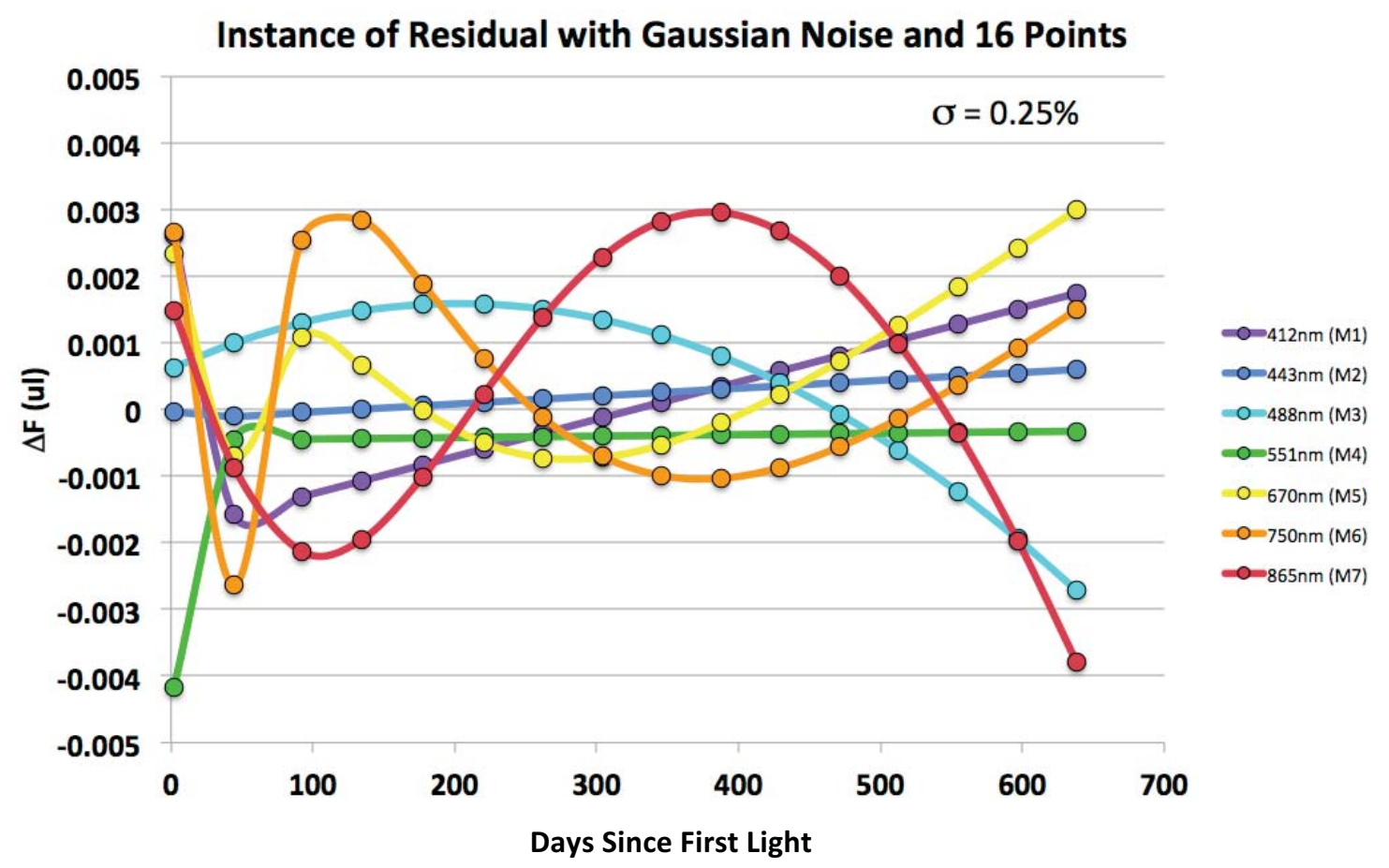

Figure 4 - Example modeled spurious trends. Results from a single trial of a Monte Carlo experiment. In this case only 16 points were generated, which is coincidentally around the number of lunar measurements the can be taken in two years time. The noise model used was based only on Gaussian noise with a standard deviation of $0.25 \%$. The modeled spurious trends are auto-correlated, even though the input noise model was not auto-correlated at all.

trend) is affected at the $0.1 \%$ level. The total influence of the white noise and seasonal variation leads to a spurious trend in calibration at the 0.1 to $0.15 \%$ level for all VisNIR bands, or up to half of the example provided in Figure 1, which is also up to half of the VIIRS calibration stability requirement threshold. Additional uncertainty will be caused by fluctuation in the green and blue bands. Influence by the red band, which is used to help the atmospheric correction in turbid and productive waters, is likely less in coastal regions and nonexistent in the clearer, deep open ocean.

\section{DISCUSSION}

The source of the season effects the VIIRS SD response trends are speculatively attributed to biases in tables used to process both the SD and the SD stability monitor (SDSM) measurements, the latter of which is discussed further, later in this section. The tables contain the SD and SDSM aperture screen transmission and SD bidirectional reflectance distribution function (BRDF) characteristics, which were obtained from laboratory measurements prior to launch. As the Sun retraces its path across the apertures each year, table biases repeat, producing a cyclic signal in the trending. Speculation that these are the likely sources arose from examination of calibration system component measurements at the DN level. These tables can be refined through further analysis of on-orbit measurements, a task which is currently underway and being conducted by the VIIRS Calibration Support Team (VCST) at GSFC. The addition information from this analysis will not only reduce the season fluctuation in the instrument responsivity monitoring, it will help substantially reduce the expected uncertainty in synoptic chlorophyll trends, likely reducing it by more than half.

In addition, an algorithmic error was recently discovered in the NOAA operational software that processes calibration data from the spacecraft and is also currently being used by NASA teams. Through a mixing of incompatible time reference systems, small errors were introduced into the solar position vector, which is used to help determine the solar irradiance on the SD and determine the position the Sun in the SD and SDSM apertures. Correction to this error is also expected to remove additional seasonal and possible secular variation in the SD calibration trends. 
As the same functional fit trending techniques are applied to both MODIS and SeaWiFS, the Chl $a$ trends from those instruments are subject to similar uncertainty constraints, although the causal details may differ. Further analysis of the noise in fit residuals for those missions could indicate much about the uncertainty in their much longer Chl $a$ trends. In general, a more detailed study is in order for VIIRS and the heritage instruments, carefully quantifying calibration trend uncertainty and following the propagation of the uncertainty from all input bands to all standard NASA derived data products. This is necessary to facilitate proper studies of climatic trends in oceanic phytoplankton activity. In addition, there are other potential sources of time dependent uncertainty that should also be explored for VIIRS, and possibly heritage instruments also. These are discussed briefly in the following subsections.

\section{Extrapolation Uncertainty}

NASA's current methodology involves trending the SD measurements periodically and offline from the operational data production. SD trends must be extrapolated into the future to facilitate forward processing. Thus, until the ocean color data products are reprocessed, all new data products are based to some degree on extrapolation of the calibration trend. Thus, any possible systematic effects of this extrapolation should be quantified and reported for forward processing after each new reprocessing version of the data is released. Future comparisons will be needed between trends fitted to the entire existing SD calibration time series and trends based on a fit of only an initial portion of that time series followed by extrapolation of the function form fit. Repeating this comparison for fits over increasing longer segments of the SD time series would provide a series of biases associated with the extrapolation as a function of the time since last reprocessing.

\section{Solar Diffuser Stability Monitor (SDSM)}

It is well know that the SD, as a reference, is not stable optically. UV light degrades the prelaunch (relatively flat) reflectance spectrum of the Spectralon ${ }^{\mathrm{TM}}$ panel to be increasingly less reflective in the blue (i.e., the SD is yellowing with time). To monitor and adjust for this effect, the SDSM is used to measure the ratio of the SD measurements to measurements of the sun through a screen. Relative changes in this ratio from first light is used to adjust the SD trending, especially for the blue bands, which are mostly affected by the SD yellowing. The uncertainty associated with ability of the SDSM to account changes to the SD should be quantified. Further work will be needed to determine the size of any spurious trend that could be introduced into the SDSM correction, and subsequently the SD calibration trend, and then eventually propagated to the chlorophyll trends. For instance, the coating in the SDSM integrating sphere that is used to observe the SD is also yellowing and could thus cause an overestimate of the SD yellowing. Also, the out-ofband (OOB) response of SDSM detectors are rapidly losing responsivity in the NIR because ambient ionizing radiation in the space environment, which is changing the SDSM spectral response characteristics.

\section{Relative Spectral Response Changes}

Because of the degradation of the instrument response in the red to SWIR regions of the spectrum over time, it follows that the relative spectral response of the instrument is also changing. In short, each band is becoming slightly, relatively more responsive in the blue end of the spectrum with time. Some OOB effects, such the known large light-leak in the $412 \mathrm{~nm}$ band, are decreasing. Compensation for OOB effects that are used for calibration is based on prelaunch characterization of the VIIRS instrument and is static. Therefore, an uncorrected, changing bias in calibration stemming from a time-dependent OOB response could contribute a small spurious trend to the TOA measurements. An investigation of this effect is underway by the authors, who have already determined that this mostly affects the $412 \mathrm{~nm}$ band, causing a change in the TOA radiance measurement of about $0.3 \%$ at present and that most of this change occurred during 2012. As this band is not used in the OC3 algorithm, its influence on the standard chlorophyll product is inconsequential. The blue and green bands are predicted to drift less than $0.1 \%$. The NIR bands are subject to a more significant change, but still at just under $0.2 \%$. But given that these increasing biases are positive for both NIR bands, the impact to the Rayleigh-subtracted band ratio used to compute the aerosol radiance is probably very small. Nonetheless, the impact of the total effect of spectral response change for all bands should be quantified.

It is also possible that this effort will benefit heritage missions, as spectral response change may not be specific to S-NPP VIIRS. Change in the instrument responsivity with time across all bands has typically not observed to be spectrally flat for either SeaWiFS or MODIS, which strongly suggests that the instrument OOB characteristics could have also changed for these instruments. 


\section{Polarization Response Changes}

To a lesser degree than MODIS, each band of the VIIRS instrument will record slightly different radiance values $(<2 \%)$ for two objects with the same radiance, but where the polarization state of the measured light is different. Light measured above the atmosphere is partially polarized by molecular scattering, the resulting degree of polarization being up to $60-70 \%$, depending on wavelength, viewing, and illumination angles. This causes a bias that must be corrected to accurately obtain $R_{r s}(\lambda)$. The response of the instrument to polarization state is characterized under laboratory conditions using a light source and a polarizer set a several different angles and the resulting characterization information is used to correct for biases in the on-orbit radiometry [18].

A concern has been recently raised amongst NASA and NOAA analysts regarding the polarization nature of the mirror contamination of S-NPP VIIRS. It known that VIIRS is changing in spectral response as a result of the mirror contamination. But, could VIIRS possibly be also changing its polarization characteristics over time? This effect would show up as a changing scan-angle dependent bias appearing over the day-lit side of each orbit. Further work to identify such variations should be part of future work.

\section{Counts-to-Radiance Converse}

Tracking changes in the instrument response with time using the SD assumes that the underlying counts-to-radiance conversion is initially and continues to be based on a linear relationship between output digital numbers (DN) and input radiance levels in geophysical units. The SD trend provides a factor, as a function of time, by which the counts-toradiance conversion is multiplied to adjust for changes in instrument response. However, it is known that some small non-linearity in the relationship between DN and input radiance exists. Furthermore, the SD provides an adjustment based on a very limited range of radiances. Therefore, as the correction from the SD trend increases, biases over other radiance levels could also grow. Because the counts-to-radiance conversion differ from detector to detector, these growing biases may also increase differently from detector to detector, appearing as gradual increases in striping in the satellite imagery over time. Other changes in the radiometric characteristics could contribute a spurious trend in the data, including but not limited to, the dark count or the radiance level of the gain switch point (pertaining to SeaWiFS and VIIRS)

\section{CONCLUSION}

An initial evaluation trend uncertainty in the VIIRS instrument responsitivity tracking was conducted. The tracking methodology was based on the standard approach used by NASA (i.e., nonlinear functional fits) for VIIRS, MODIS, and SeaWiFS. Monte Carlo experiments used residual characteristics to model SD trend noise and estimate the size of modeled spurious trends introduced by the nonlinear fitting process. The size of these spurious trends, for all bands, was not surprisingly similar to the residual noise amplitude. But these spurious trends by nature are highly auto-correlated and can lead to false variation in chlorophyll $a$ concentration data product. Tying the modeled spurious calibration trends to fluctuations in the chlorophyll $a$ concentrations suggests significant false variation in the latter could exist on the level of a few percent over the course of the VIIRS mission. Anti-correlated NIR contributions were shown to possibly affect coastal and open waters differently, possibly leading to misinterpretation of ecological responses to climate. Additional variation would be expected from spurious trends in the visible bands. Improvement to operational look-up tables for the SD and SDSM characteristics and to the code used for determining the solar position vector are expected to reduce spurious trends in VIIRS calibration. Further analysis should be conducted to evaluate the total influence of calibration trend uncertainty in all bands to all ocean color derived products. Other sources of time dependent variation should also be investigated. This and continued analysis of trend uncertainty will be crucial to understand the epistemological validity of statements based on satellite data that are made about synoptic climatic effects in ocean biology.

\section{REFERENCES}

[1] O'Reilly, J. E., Maritorena, S., Mitchell, B. G. et al., "Ocean color chlorophyll algorithms for SeaWiFS,” Journal of Geophysical Research, 103, 24937-24953 (1998).

[2] Boyce, D. G., Dowd, M., Lewis, M. R. et al., "Estimating global chlorophyll changes over the past century," Progress in Oceanography, (2014). 
[3] Boyce, D. G., Lewis, M. R., and Worm, B., "Global phytoplankton decline over the past century," Nature, 466(7306), 591-6 (2010).

[4] Gregg, W. W., and Conkright, M. E., "Decadal changes in global ocean chlorophyll," Geophysical Research Letters, 29(15), 1730-1734 (2002).

[5] Polovina, J. J., Howell, E. A., and Abecassis, M., “Ocean's least productive waters are expanding," Geophysical Research Letters, 35(3), (2008).

[6] McClain, C. R., "A decade of satellite ocean color observations," Ann Rev Mar Sci, 1, 19-42 (2009).

[7] Vantrepotte, V., and Mélin, F., "Inter-annual variations in the SeaWiFS global chlorophyll a concentration (19972007),” Deep Sea Research Part I: Oceanographic Research Papers, 58(4), 429-441 (2011).

[8] Kahru, M., and Mitchell, B. G., "Ocean color reveals increased blooms in various parts of the world," EOS Transactions of the American Geophysical Union, 89, 170 (2008).

[9] Behrenfeld, M. J., O'Malley, R. T., Siegel, D. A. et al., "Climate-driven trends in contemporary ocean productivity," Nature, 444(7120), 752-5 (2006).

[10] Beaulieu, C., Henson, S. A., Sarmiento, J. L. et al., "Factors challenging our ability to detect long-term trends in ocean chlorophyll," Biogeosciences, 10(4), 2711-2724 (2013).

[11] Siegel, D. A., Behrenfeld, M. J., Maritorena, S. et al., "Regional to global assessments of phytoplankton dynamics from the SeaWiFS mission," Remote Sensing of Environment, 135, 77-91 (2013).

[12] Vantrepotte, V., and Me'lin, F., "Temporal variability of 10-year global SeaWiFS time-series of phytoplankton chlorophyll a concentration," Journal of Marine Science, 66, 1547-1556 (2014).

[13] Wernand, M. R., van der Woerd, H. J., and Gieskes, W. W., "Trends in ocean colour and chlorophyll concentration from 1889 to 2000, worldwide," PLoS One, 8(6), e63766 (2013).

[14] Henson, S. A., Sarmiento, J. L., Dunne, J. P. et al., "Detection of anthropogenic climate change in satellite records of ocean chlorophyll and productivity," Biogeosciences, 7, 621-640 (2010).

[15] Yoder, J. A., Kennelly, M. A., Doney, S. C. et al., "Are trends in SeaWiFS chlorophyll time-series unusual relative to historic variability," Acta Oceanologica Sinica, 29(2), 1-4 (2010).

[16] Turpie, K. R., McClain, C. R., Esaias, W. E. et al., [Sensitivity of ocean color remote sensing from space to calibration errors] NASA Goddard Space Flight Center, Greenbelt, Md, (2009).

[17] Eplee, R. E., Jr., Turpie, K. R., Meister, G. et al., "A synthesis of VIIRS solar and lunar calibrations,", in Earth Observing Systems XVIII J.J. . 8855, 88661L.

[18] Meister, G., Kwiatkowska, E. J., Franz, B. A. et al., "Moderate-resolution imaging spectroradiometer ocean color polarization correction," Applied Optics, 44(26), 5524-5535 (2005). 